\title{
Multimodel Predictive Control Approach for UAV Formation Flight
}

\author{
Chang-jian Ru, ${ }^{1}$ Rui-xuan Wei, ${ }^{1}$ Ying-ying Wang, ${ }^{1}$ and Jun Che ${ }^{2}$ \\ ${ }^{1}$ Air Force Engineering University, Xi'an 710038, China \\ ${ }^{2}$ Science and Technology on Aircraft Control Laboratory, FACRI, Xian 710065, China \\ Correspondence should be addressed to Chang-jian Ru; ruchangjian1986@gmail.com
}

Received 20 December 2013; Revised 10 March 2014; Accepted 25 March 2014; Published 6 May 2014

Academic Editor: Leo Chen

Copyright (c) 2014 Chang-jian Ru et al. This is an open access article distributed under the Creative Commons Attribution License, which permits unrestricted use, distribution, and reproduction in any medium, provided the original work is properly cited.

\begin{abstract}
Formation flight problem is the most important and interesting problem of multiple UAVs (unmanned aerial vehicles) cooperative control. In this paper, a novel approach for UAV formation flight based on multimodel predictive control is designed. Firstly, the state equation of relative motion is obtained and then discretized. By the geometrical method, the characteristic points of state are determined. Afterwards, based on the linearization technique, the standard linear discrete model is obtained at each characteristic state point. Then, weighted model set is proposed using the idea of T-S (Takagi-Sugeno) fuzzy control and the predictive control is carried out based on the multimodel method. Finally, to verify the performance of the proposed method, two different simulation scenarios are performed.
\end{abstract}

\section{Introduction}

In recent years, as an advanced system with high autonomy, UAVs have been widely applied in the fields of both civilian and military. When a single UAV accomplishes tasks individually, it will be more likely to reduce mission success, due to its limited information accessing ability. In comparison, multiple UAVs collaborating with each other maintain a certain formation during the flight, which provides them with full access to environmental information, increases resistance to external attack capability, improves working efficiency and robustness of the system, and so forth, so it has attracted wide attention [1-3]. Formation flight is an important aspect of multiple UAVs cooperative control. When the formation shape maintains or changes according to the mission requirements, it is necessary to control the relative position, attitude, and speed between UAVs, and so forth. However, UAV's control system is a nonlinear coupling system, coupled with complex operational environment constraints, putting forward higher design requirement for formation controller. So it is essential to propose an effective control strategy to solve those problems.
Model predictive control (MPC) method is put forward by some scholars. Here, several typical researches are presented. A hierarchical approach and a set of MPC strategies for the UAV formation are proposed in [4], where obstacle and collision avoidance constraints are taken into account. A distributed collision-free formation flight control law in the framework of nonlinear model predictive control is designed in [5]. In [6], a dual mode MPC method is used for formation control. To guarantee the stability, the dual mode controller must switch from an MPC control to a terminal state controller. A simple nonlinear model predictive control (NMPC) formulation is used to adequately address the terrain avoidance problem, as presented in [7]. An online nonlinear model predictive control framework is used for the trajectory tracking of autonomous vehicles in [8], where a bicycle model is used for the prediction of future states in the NMPC framework. The validation of a formation flight control technique with obstacle avoidance capability based on nonlinear model predictive algorithms is proposed in [9].

The nonlinear model predictive control method provides an effective means to solve the control problem of nonlinear systems $[10,11]$. Because the close relative distance between 


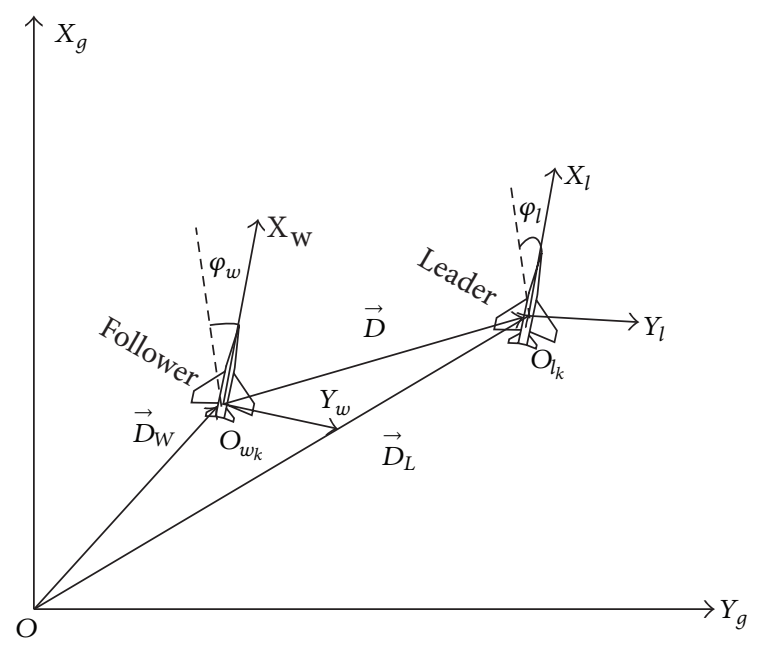

FIGURE 1: The position relationship between two vehicles.

the UAVs may lead to collision; thus, it requires higher control accuracy. However, stair-like MPC uses the way of constraining the variation of the future control quantity, which restricts the maneuverability of the vehicle and is prone to causing collision between the UAVs due to the overshoot problem [12]. So it is necessary to adopt a new predictive control method to achieve the formation flight control. For some more complex systems, the multimodel control method has stronger robustness and higher control accuracy under certain conditions [13]. Besides, multimodel control method can provide the nonlinear system with transparent model and controller, facilitating the system analysis. Compared with other nonlinear global strategies, the multimodel control method cannot greatly reduce computational complexity, but the model and structure of controller are more suitable for online adjustments and learning algorithm [14], so multiple model-based predictive control can be used to solve UAV formation control problem.

This paper is organized as follows. In Section 2, the discrete relative motion equations for UAV formation are established. In Section 3, a multiple models-based predictive control approach is used to design controller of the formation. Simulation results are given in Section 4. Finally, Section 5 concludes the paper.

\section{UAV Formation Flight Control Model}

2.1. Kinematics Model of UAV Formation Flight Control. Assume that during the formation flight, an UAV is flying horizontally and has no sideslip. In the geographic coordinate system, the relationship between the position vectors of leader UAV (leader) and follower UAV (follower) is shown in Figure 1.

From Figure 1, it is easy to obtain the following equation:

$$
\vec{D}_{L}=\vec{D}_{W}+\vec{D}
$$

where, $\vec{D}_{L}, \vec{D}_{W}$ are displacement vectors of two vehicles and $\vec{D}$ is the relative displacement vectors between two vehicles.
Differentiating (1), one can obtain

$$
\frac{d \vec{D}_{L}}{d t}=\frac{d \vec{D}_{W}}{d t}+\frac{d \vec{D}}{d t} .
$$

According to the relationship between the moving coordinate system, one can easily obtain

$$
\frac{d \vec{D}_{L}}{d t}=\frac{\delta \vec{D}_{W}}{\delta t}+\omega_{w} \times \vec{D}
$$

where $\omega_{w}$ is the yaw angular rate.

Since the vehicle is supposed to fly horizontally, the equation of motion will be

$$
\begin{aligned}
& \dot{x}_{i}=v_{i} \cos \left(\varphi_{i}\right), \\
& \dot{y}_{i}=v_{i} \sin \left(\varphi_{i}\right), \\
& \dot{\varphi}_{i}=\omega_{i}, \quad i=l, w,
\end{aligned}
$$

where the subscript $l$ and $w$ denote leader UAV and follower UAV, respectively.

Combing (3) and (4), the relative motion equation of two vehicles can be obtained as

$$
v_{l}\left[\begin{array}{c}
\cos \varphi_{l} \\
\sin \varphi_{l}
\end{array}\right]=v_{w}\left[\begin{array}{c}
\cos \varphi_{w} \\
\sin \varphi_{w}
\end{array}\right]+C_{w}^{L}\left(\left[\begin{array}{c}
\dot{x}_{d} \\
\dot{y}_{d}
\end{array}\right]+\left[\begin{array}{c}
-\omega_{w} \cdot y_{d} \\
\omega_{w} \cdot x_{d}
\end{array}\right]\right),
$$

where $x_{d}$ and $y_{d}$ are $X$-axis value and $Y$-axis value of the distance between two vehicles in the tack coordinates of leader $\mathrm{UAV}$, respectively, and $C_{w}^{L}$ is the coordinate transformation matrix.

Then, carry on the translational process, and one can obtain

$$
\left[\begin{array}{c}
\dot{x}_{d} \\
\dot{y}_{d}
\end{array}\right]=C_{L}^{W}\left(v_{l}\left[\begin{array}{c}
\cos \varphi_{l} \\
\sin \varphi_{l}
\end{array}\right]-v_{w}\left[\begin{array}{c}
\cos \varphi_{w} \\
\sin \varphi_{w}
\end{array}\right]\right)-\left[\begin{array}{c}
-\omega_{w} \cdot y_{d} \\
\omega_{w} \cdot x_{d}
\end{array}\right],
$$

where

$$
\begin{gathered}
C_{L}^{W} v_{l}\left[\begin{array}{c}
\cos \varphi_{l} \\
\sin \varphi_{l}
\end{array}\right]=v_{l}\left[\begin{array}{c}
\cos \varphi_{e} \\
\sin \varphi_{e}
\end{array}\right], \\
C_{L}^{W} v_{w}\left[\begin{array}{c}
\cos \varphi_{w} \\
\sin \varphi_{w}
\end{array}\right]=\left[\begin{array}{c}
v_{w} \\
0
\end{array}\right] .
\end{gathered}
$$

So (6) can be written as follows:

$$
\left[\begin{array}{c}
\dot{x}_{d} \\
\dot{y}_{d}
\end{array}\right]=v_{l}\left[\begin{array}{c}
\cos \left(\varphi_{l}-\varphi_{w}\right) \\
\sin \left(\varphi_{l}-\varphi_{w}\right)
\end{array}\right]-\left[\begin{array}{c}
v_{w} \\
0
\end{array}\right]-\left[\begin{array}{c}
-\omega_{w} \cdot y_{d} \\
\omega_{w} \cdot x_{d}
\end{array}\right] .
$$

Additionally, there is

$$
\dot{\varphi}_{w}=\omega_{w}
$$

Combing (8) and (9), we can obtain

$$
\begin{aligned}
& \dot{x}_{d}=v_{l} \cos \left(\varphi_{l}-\varphi_{w}\right)-v_{w}+\omega_{w} \cdot y_{d}, \\
& \dot{y}_{d}=v_{l} \sin \left(\varphi_{l}-\varphi_{w}\right)-\omega_{w} \cdot x_{d}, \\
& \dot{\varphi}_{w}=\omega_{w} .
\end{aligned}
$$


Then, (10) can also be written as the state equations which is shown as follows

$$
\left[\begin{array}{c}
\dot{x}_{d} \\
\dot{y}_{d} \\
\dot{\varphi}_{w}
\end{array}\right]=\left[\begin{array}{cc}
-1 & y_{d} \\
0 & -x_{d} \\
0 & 1
\end{array}\right]\left[\begin{array}{c}
v_{w} \\
\omega_{w}
\end{array}\right]+\left[\begin{array}{c}
v_{l} \cos \left(\varphi_{l}-\varphi_{w}\right) \\
v_{l} \sin \left(\varphi_{l}-\varphi_{w}\right) \\
0
\end{array}\right] .
$$

The output equation is

$$
\begin{aligned}
& y_{1}=x_{d}, \\
& y_{2}=y_{d} .
\end{aligned}
$$

2.2. Discrete Model of UAV Formation Movement and Its Predictive Control Analysis. In the previous section, the state equation of formation control is obtained. Here, discretize this equation and the following equation can be obtained

$$
\begin{aligned}
{\left[\begin{array}{c}
x_{d}(k+1) \\
y_{d}(k+1) \\
\varphi_{w}(k+1)
\end{array}\right]=} & {\left[\begin{array}{l}
x_{d}(k) \\
y_{d}(k) \\
\varphi_{w}(k)
\end{array}\right]+\left[\begin{array}{cc}
-1 & y_{d}(k) \\
0 & -x_{d}(k) \\
0 & 1
\end{array}\right]\left[\begin{array}{c}
v_{w}(k) \\
\omega_{w}(k)
\end{array}\right] \Delta T } \\
& +\left[\begin{array}{c}
v_{l}(k) \cos \left(\varphi_{l}(k)-\varphi_{w}(k)\right) \\
v_{l}(k) \sin \left(\varphi_{l}(k)-\varphi_{w}(k)\right) \\
0
\end{array}\right] \Delta T .
\end{aligned}
$$

Generally, the sampling periodic time is short during receding optimization process, so the velocity and yaw angle of leader UAV can be considered constant in sampling period [15], which means in a short sampling period, there is

$$
\begin{gathered}
v_{l}(k+i)=v_{l}(k+i-1)=v_{l}(k), \\
\varphi_{l}(k+i)=\varphi_{l}(k+i-1)=\varphi_{l}(k) .
\end{gathered}
$$

According to these two equations, the predicted value of outputs will be obtained as follows:

$$
\begin{aligned}
& x_{d_{p}}(k+1), x_{d_{p}}(k+2), \ldots, x_{d_{p}}(k+N), \\
& y_{d_{p}}(k+1), y_{d_{p}}(k+2), \ldots, y_{d_{p}}(k+N) .
\end{aligned}
$$

Since at time $k, y_{d}(k), v_{l}(k), \varphi_{w}(k), \varphi_{l}(k)$ are known, the future output values of formation flight control are $x_{d p}(k+$ $1), x_{d_{p}}(k+2), \ldots, x_{d_{p}}(k+N-1), x_{d_{p}}(k+N)$, and $y_{d_{p}}(k+$ $1), y_{d_{p}}(k+2), \ldots, y_{d_{p}}(k+N-1), y_{d_{p}}(k+N)$, and these outputs are only the function of the future control quantities $v_{w}(k), v_{w}(k+1), \ldots, v_{w}(k+N-1)$ and $\omega_{w}(k), \omega_{w}(k+$ $1), \ldots, \omega_{w}(k+N-1)$. Obviously, this function is a multi-inputmultioutput nonlinear control problem. So, these values can be obtained using the receding optimization algorithm. Then, using $v_{w}(k)$ and $\omega_{w}(k)$ as outputs of control quantity, and carry out the receding optimization algorithm in sequence, the input of control quantity in the next time can be obtained.

According to Section 1, it can be known that for the problem of UAV formation flight, nonlinear model predictive control and fuzzy stair-like predictive control have some limitations, but the multimodel control method has stronger robustness and higher control accuracy, the final predictive model of which is linear, so the receding optimization problem can be changed from general nonlinear optimization problem to a linear quadratic optimization problem. Since the linear quadratic optimization has faster computation than the ordinary nonlinear optimization, the multiple models can greatly improve real-time of receding optimization [16]. Therefore, multiple model-based predictive control approach is used to design the controller of UAV formation flight.

\section{Predictive Control for UAV Formation Based on Multimodel Approach}

The basic principle of the multimodel control method is that nearby the different characteristics of nonlinear systems, different linear model is used to describe this nonlinear system, and each linear model only describes a part of nonlinear system dynamics. The multiple linearization models are used to approximate the nonlinear system in its entire operating range, and the controller is designed based on each linearization model. These controllers are combined together to constitute a multimodel controller in some way. Finally, the control of entire nonlinear system can be achieved through coordinated control between multiple linearization models. The basic steps of this method can be summarized in four steps: (1) acquisition of the model set; (2) local linearization of the model set; (3) establishment of the controller set; (4) combination of the model set.

Similarly, the controller design of UAV formation flight can also include these steps, the flow chart of which is shown in Figure 2. The details of controller design will be presented in the following parts using the multimodel prediction method.

3.1. Determination of the State Characteristic Points of Formation Control Model. According to the basic principles of multimodel predictive control, the characteristic state points of the nonlinear model must be obtained first before obtaining multiple models. Characteristic state points and their region are determined using methods in $[15,17]$. As is shown in Figure 3, the horizontal axis and vertical axis denote time and trajectory, respectively.

The basic idea is as follows.

Determine the first characteristic state points, then compute the error between reference trajectory tangent through the characteristic point and reference trajectory and compare the error with the maximum permissible error. If it is greater than the maximum permissible error, redetermine the characteristic point to get the next characteristic state point, and calculate repeatedly until the last characteristic state point is obtained; thus, the characteristic state points of nonlinear system and their applicable region can be determined. So the linearization model set can be obtained through the linearization process at the characteristic state point for each region.

For UAV formation control, the characteristic state points are determined as follows.

Assuming that the initial distance between two vehicles are $x_{d}(0)$ and $y_{d}(0)$, respectively, when UAV formation control is carried out, so the desired distances of UAV formation 


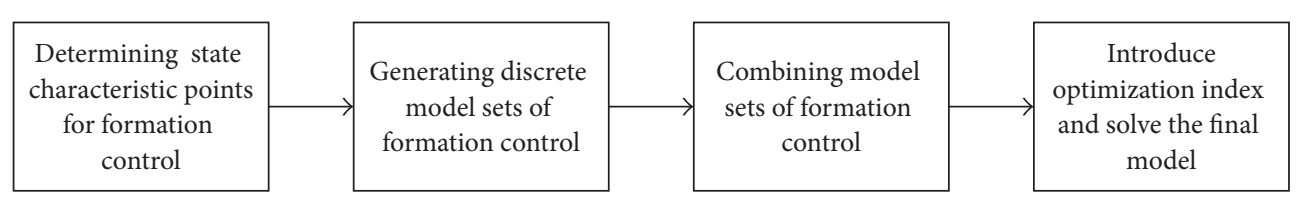

FIGURE 2: The flow chart of UAV formation flight controller design.

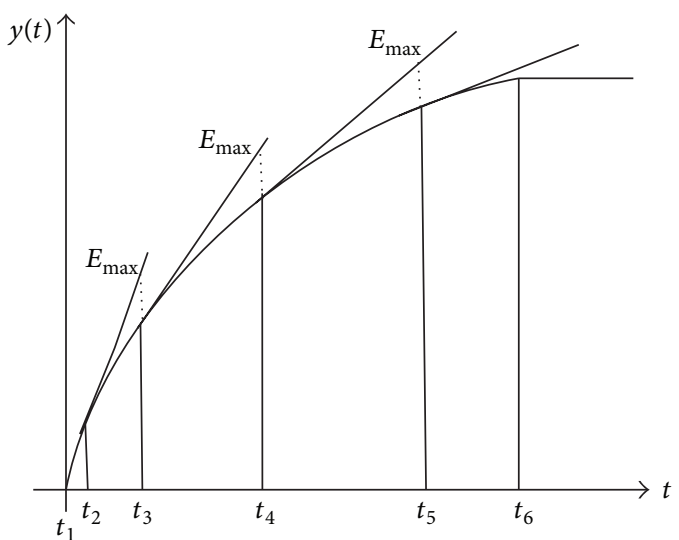

FIGURE 3: Description of tangent error.

are $x_{c}$ and $y_{c}$. Assuming that the expected time arrival at the desired value of $X$-axis and $Y$-axis is the same, the reference trajectory of UAV formation can be obtained as follows:

$$
\begin{aligned}
& y_{1 r}(t)=\xi^{t} x_{d}(0)+\left(1-\xi^{t}\right) \cdot x_{c}, \\
& y_{2 r}(t)=\xi^{t} y_{d}(0)+\left(1-\xi^{t}\right) \cdot y_{c} .
\end{aligned}
$$

Since there are two outputs, generating two reference trajectories, the algorithm above cannot be applied directly. But, because the expected arrival time to the desired value is the same, the reference trajectory of an output can be used to determine the characteristic point of state. Assume that the reference trajectory of relative position on the $X$-axis in the track coordinate system is used to determine characteristic point of the state.

At the characteristic state points, there is

$$
\dot{x}_{d}=0, \quad \dot{y}_{d}=0, \quad \dot{\varphi}_{w}=0 \text {. }
$$

Assume that $i$ th characteristic state point is $\left(V_{w}^{i}, \omega_{w}^{i}, x_{d}^{i}\right.$, $\left.y_{d}^{i}, \varphi_{d}^{i}\right)$, then there will be the following equation:

$$
\left[\begin{array}{cc}
-1 & y_{d}^{i} \\
0 & -x_{d}^{i} \\
0 & 1
\end{array}\right]\left[\begin{array}{c}
V_{w}^{i} \\
\omega_{w}^{i}
\end{array}\right]+\left[\begin{array}{c}
V_{l} \cos \left(\varphi_{l}-\varphi_{w}^{i}\right) \\
V_{l} \sin \left(\varphi_{l}-\varphi_{w}^{i}\right) \\
0
\end{array}\right]=\left[\begin{array}{l}
0 \\
0 \\
0
\end{array}\right] .
$$

Meanwhile, there is

$$
x_{d}^{i}=y_{1 r}(i), \quad y_{d}^{i}=y_{2 r}(i) .
$$

Solve (18) and (19), then we can obtain

$$
\begin{gathered}
\omega_{w}^{i}=0, \quad \varphi_{w}^{i}=\varphi_{l}, \quad v_{w}^{i}=v_{l}, \\
x_{d}^{i}=y_{1 r}(i), \quad y_{d}^{i}=y_{2 r}(i) .
\end{gathered}
$$

Assuming the initial point is as the first characteristic state point, then the state point value will be

$$
\begin{gathered}
\omega_{w}^{1}=0, \quad \varphi_{w}^{1}=\varphi_{l}, \quad v_{w}^{1}=v_{l}, \\
x_{d}^{1}=y_{1 r}(0), \quad y_{d}^{1}=y_{2 r}(0) .
\end{gathered}
$$

Then, the equation of the tangent is

$$
y_{1 k}(t)=\dot{y}_{1 r}(t) t+x_{d}(0) .
$$

Afterward, determine the maximum permissible error $E_{\text {max }}$, and the time $t_{2}$ corresponding to the second characteristic point can be obtained using the method above. Then, the state point can be obtained as follows

$$
\begin{gathered}
\omega_{w}^{2}=0, \quad \varphi_{w}^{2}=\varphi_{l}, \quad v_{w}^{2}=v_{l}, \\
x_{d}^{2}=x_{d}\left(t_{2}\right), \quad y_{d}^{2}=y_{d}\left(t_{2}\right) .
\end{gathered}
$$

So the tangent equation is

$$
y_{1 k}(t)=\dot{y}_{1 r}(t)\left(t-t_{2}\right)+x_{d}\left(t_{2}\right) \text {. }
$$

By repeating these procedures above, time $t_{i}$ corresponding to the characteristic point in the region of multimodel for formation can be obtained, and at the same time the characteristic state point corresponding to the time $t_{i}$ can be also obtained:

$$
\begin{gathered}
\omega_{w}^{i}=0, \quad \varphi_{w}^{i}=\varphi_{l}, \quad v_{w}^{i}=v_{l}, \\
x_{d}^{i}=x_{d}\left(t_{i}\right), \quad y_{d}^{i}=y_{d}\left(t_{i}\right) .
\end{gathered}
$$

Calculate until the last characteristic state point is obtained, and then the computation will be terminated.

\subsection{Generation of Discrete Model Sets for Formation Control.} After obtaining the characteristic state points, carry on linearization at different discrete model sets of formation. Here, linearization can be realized through the following methods.

Consider nonlinear systems as described in the form of discrete-time dynamic equations:

$$
\begin{gathered}
x(k+1)=f(x(k), u(k)), \\
y(k)=g(x(k), u(k)) .
\end{gathered}
$$


The system has $m$ different characteristic state points. $f(x(k), u(k))$ and $g(x(k), u(k))$ have the first continuous partial derivative. If system is linearized at each characteristic state point, the standard discrete state-space model of $m$ linear models of the original system is obtained as follows:

$$
\begin{gathered}
x(k+1)=A_{i} x(k)+B_{i} u(k)-\alpha_{i} . \\
y(k)=C_{i} x(k)+D_{i} u(k)-\beta_{i},
\end{gathered}
$$

where

$$
\begin{gathered}
A_{i}=\left.\frac{\partial f}{\partial x}\right|_{\left(x_{i}, u_{i}\right)}, \quad B_{i}=\left.\frac{\partial f}{\partial u}\right|_{\left(x_{i}, u_{i}\right)}, \quad C_{i}=\left.\frac{\partial g}{\partial x}\right|_{\left(x_{i}, u_{i}\right)}, \\
D_{i}=\left.\frac{\partial g}{\partial u}\right|_{\left(x_{i}, u_{i}\right)}, \quad \alpha_{i}=\left.\frac{\partial f}{\partial x}\right|_{\left(x_{i}, u_{i}\right)} x_{i}+\left.\frac{\partial f}{\partial u}\right|_{\left(x_{i}, u_{i}\right)} u_{i}-x_{i}, \\
\alpha_{i}=\left.\frac{\partial g}{\partial x}\right|_{\left(x_{i}, u_{i}\right)} x_{i}+\left.\frac{\partial g}{\partial u}\right|_{\left(x_{i}, u_{i}\right)} u_{i}-y_{i} .
\end{gathered}
$$

Here, $m$ linearized models constitute the linearized multimodel presentation of the original system.

For the UAV formation flying control, the characteristic state points are shown as

$$
\begin{gathered}
\omega_{w}^{i}=0, \quad \varphi_{w}^{i}=\varphi_{l}, \quad v_{w}^{i}=v_{l}, \\
x_{d}^{i}=x_{d}\left(t_{i}\right), \quad y_{d}^{i}=y_{d}\left(t_{i}\right) .
\end{gathered}
$$

And it has the following expression:

$$
\begin{gathered}
A_{i}=\left.\frac{\partial f}{\partial x}\right|_{\left(x_{i}, u_{i}\right)}=\left[\begin{array}{ccc}
1 & 0 & 0 \\
0 & 1 & -v_{l}(k) \Delta T \\
0 & 0 & 1
\end{array}\right], \\
B_{i}=\left.\frac{\partial f}{\partial u}\right|_{\left(x_{i}, u_{i}\right)}=\left[\begin{array}{cc}
-1 & y_{d}\left(t_{i}\right) \\
0 & -x_{d}\left(t_{i}\right) \\
0 & 1
\end{array}\right] \Delta T \\
\alpha_{i}=\left.\frac{\partial f}{\partial x}\right|_{\left(x_{i}, u_{i}\right)} x_{i}+\left.\frac{\partial f}{\partial u}\right|_{\left(x_{i}, u_{i}\right)} u_{i}-x_{i}=\left[\begin{array}{c}
-v_{l}(k) \Delta T \\
-v_{l}(k) \varphi_{l}(k) \Delta T \\
0
\end{array}\right] .
\end{gathered}
$$

Since outputs are linear, $C_{i}, D_{i}$ and $\beta_{i}$ will not be solved. Thus, the linearization equation at characteristic state point will be

$$
\begin{aligned}
& {\left[\begin{array}{l}
x_{d}(k+1) \\
y_{d}(k+1) \\
\varphi_{d}(k+1)
\end{array}\right]} \\
& =\left[\begin{array}{ccc}
1 & 0 & 0 \\
0 & 1 & -v_{l}(k) \Delta T \\
0 & 0 & 1
\end{array}\right]\left[\begin{array}{l}
x_{d}(k) \\
y_{d}(k) \\
\varphi_{d}(k)
\end{array}\right] \\
& \quad+\left[\begin{array}{cc}
-1 & y_{d}\left(t_{i}\right) \\
0 & x_{d}\left(t_{i}\right) \\
0 & 1
\end{array}\right] \Delta T\left[\begin{array}{c}
v_{w}(k) \\
\omega_{w}(k)
\end{array}\right]-\left[\begin{array}{c}
-v_{l}(k) \Delta T \\
-v_{l}(k) \varphi_{l}(k) \Delta T \\
0
\end{array}\right] .
\end{aligned}
$$

So for different characteristic state points, the linear model for UAV formation control at different horizons can be obtained, realizing the acquisition of model sets for UAV formation. And these models are denoted as $M(1), M(2), \ldots, M(S)$.

\subsection{Combined Method of Model Sets for Formation Flight} Control. The characteristic state points are obtained using the method above. When the error reaches the maximum value, switch to the new model, which ensures the maximum error value between the predictive trajectory and the reference trajectory. Thus, the determination of model region can be realized. It can be seen that the applicable range of desired model is divided based on the time region, so different sampling points have different models. But the predictive control is based on the future time region. So in this paper, the applicable model of predictive point is judged by the time region, and then this model is used to calculate the predictive value. The judgment rules of predictive model are described as follows.

Assuming that the time corresponding to the state characteristic points is $t_{1}<t_{2}<\cdots<t_{s-1}<t_{s}$ and the predictive horizon is $[t, t+N]$, then there will be the following.

If $[t, t+N] \in\left[t_{i}, t_{i+1}\right]$, then the final predictive model of all points is $M_{T}=M_{i}$; if $[t, t+N] \in\left[t_{i}, t_{i+N}\right]$, one can judge the interval $\left[t_{h}, t_{h+1}\right]$ of all points between prediction point $t+1$ to $t+N$ in sequence and denote the model of this point as $M_{T}=M_{h}$; if $[t, t+N] \in\left[t_{s}, \infty\right)$, the final predictive model of all the points will be $M_{T}=M_{s}$.

Based on this method, we can obtain the predictive function during the future horizon to determine the optimization index. However, the boundary point of the predictive range may be closer to the next linear model, as shown in Figure 4. Sampling point $p_{1}$ may be closer to the linear model at sampling point $t_{4}$. But according to the method above, the calculation model used at the sampling point $p_{1}$ is the linear model of the state characteristic point at sampling point $t_{3}$. Sampling point $p_{2}$ may be closer to the linear model of the state characteristic point at sampling point $t_{5}$. But according to the method above, the calculation model used by the sampling point $p_{2}$ is the linear model of the state characteristic point at sampling point $t_{4}$.

So the method above may decrease the performance of the approximation capability on the boundary, and each model belonging to the model set cannot switch smoothly [18]. However, T-S fuzzy model as an intelligent control method, mainly uses fuzzy reasoning to approximate the nonlinear system. Using this method, the input space can be divided into several fuzzy subspaces, where a local linear model is established, and then the local models are combined smoothly using the membership function, forming a global fuzzy model of nonlinear function, which is ultimately identified as a linear model [19]. The predictive control method based on the T-S fuzzy model belongs to multimodel predictive control with the weighted models. Compared with the common multimodel predictive controllers with weighted models, the fuzzy weighted models have more accurate nonlinear approximation performance; switch of 


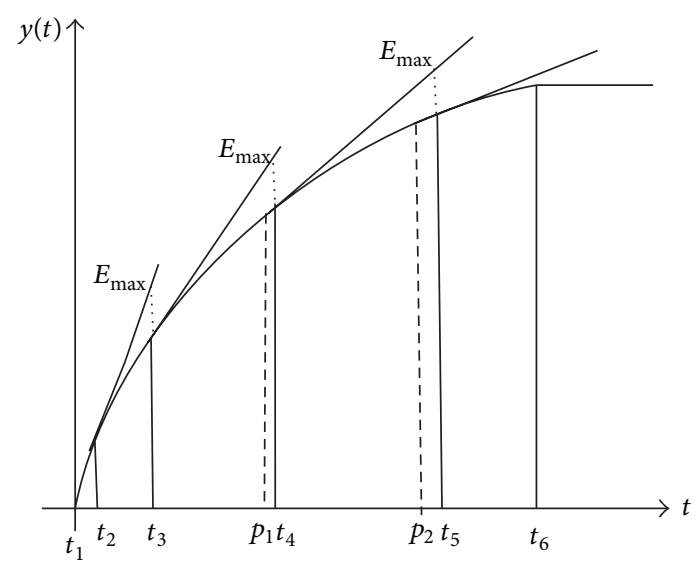

FIGURE 4: The schematic for determining model of frontier points during the predictive intervals.

the model is more smooth, and it is easier to understand $[20,21]$. So in this section, T-S fuzzy idea is adopted for the multimodel control of UAV formation flight, as is shown in Figure 5.

For each sampling point, use the error between the tangent of state characteristic point and the reference trajectory of this sampling point to calculate the membership degree. Assuming that the error between tangent of $j$ th state characteristic point and the reference trajectory at the sampling point $t$ is $E_{i}(t)$, so for the point $t+i$ in the predictive range, weighted function is as follows:

$$
\begin{gathered}
w_{j}(t+i)=e^{-\left(E_{i}(t+i) / E_{\max }\right)^{2}}, \quad-E_{\max } \leq E_{j}(t+i) \leq E_{\max }, \\
w(t+i)=0, \quad E_{j}(t+i) \leq-E_{\max } \\
\text { or } \quad E_{j}(t+i) \geq E_{\max } .
\end{gathered}
$$

This equation can ensure that the farther away from the state characteristic point the sampling point is, the lower its weighted value is. Using the weighted values, the prediction model at the sampling point $t$ is

$$
M_{t}=\frac{\sum_{i=1}^{m}\left(w_{i} M_{i}\right)}{\sum_{i=1}^{m}\left(w_{i}\right)} .
$$
there is

For the sampling points during the predictive horizon,

$$
y_{p}(t+i)=\frac{\sum_{j=1}^{m}\left(w_{j}(t+i) M_{j}\right)}{\sum_{j=1}^{m}\left(w_{j}(t+i)\right)},
$$

where

$$
\begin{gathered}
w_{j}(t+i)=e^{-\left(E_{i}(t+i) / E_{\max }\right)^{2}}, \\
-E_{\max } \leq E_{j}(t+i) \leq E_{\max }, \\
w(t+i)=0, \quad E_{j}(t+i) \leq-E_{\max } \\
\text { or } \quad E_{j}(t+i) \geq E_{\max } .
\end{gathered}
$$

Through this approach, the linear prediction function for UAV formation can be obtained as follows:

$$
\begin{aligned}
& x_{d_{p}}(k+1), x_{d_{p}}(k+2), \ldots, x_{d_{p}}(k+N), \\
& y_{d_{p}}(k+1), y_{d_{p}}(k+2), \ldots, y_{d_{p}}(k+N) .
\end{aligned}
$$

In this way, predictive outputs can change from a nonlinear function to a linear function. This nonlinear function includes $v_{w}(k), v_{w}(k+1), \ldots, v_{w}(k+N-1)$, and $\omega_{w}(k), \omega_{w}(k+$ $1), \ldots, \omega_{w}(k+N-1)$, while this linear function includes the control quantities mentioned above. Thus, the control problem will become a multi-input-multioutput linear predictive control problem.

3.4. Optimization Index for Formation and Receding Optimization Solution. During the predictive control process, the goal of receding optimization is to find a set of $v_{w}(k), v_{w}(k+$ $1), \ldots, v_{w}(k+N-1)$ and $\omega_{w}(k), \omega_{w}(k+1), \ldots, \omega_{w}(k+N-1)$, making prediction outputs at entire optimization horizon as close to the reference trajectory as possible.

Here, introduce the closed-loop

$$
\begin{aligned}
& e_{1}(t)=x_{d}(t)-x_{d_{p}}(t), \\
& e_{2}(t)=y_{d}(t)-y_{d_{p}}(t) .
\end{aligned}
$$

The open-loop predictive output can be directly compensated by the output feedback, and then the predictive value of the closed-loop model will be

$$
\begin{aligned}
& x_{d}(t+i)=x_{d p}(t+i)+e_{1}(t), \\
& y_{d}(t+i)=y_{d p}(t+i)+e_{2}(t) .
\end{aligned}
$$

In this section, there are two control objectives: the relative distances to $X$-axis and $Y$-axis. Since they have equal importance and the same unit of quantity, they are set with the same weight when designing the performance index. Thus, the performance index is defined as follows:

$$
\begin{aligned}
J=\sum_{i=1}^{N}\left[\left(y_{1 r}(t+i)-x_{d}(t+i)\right)^{2}\right. \\
\left.+\left(y_{2 r}(t+i)-y_{d}(t+i)\right)^{2}\right] \\
+\lambda_{1} \sum_{i=0}^{N-1}\left(v_{w}(t+i)-v_{w}(t)\right)^{2} \\
+\lambda_{2} \sum_{i=0}^{N-1} \omega_{w}^{2}(t+i) .
\end{aligned}
$$

Similarly, the optimization constraints of control quantity are introduced as follows:

$$
\begin{aligned}
v_{w}(k+i-1)-\Delta v & <v_{w}(k+i)<v_{w}(k+i-1)+\Delta v \\
\omega_{\min } & <\omega_{w}(k+i)<\omega_{\max } \\
v_{\min } & <v_{w}(k+i)<v_{\max }
\end{aligned}
$$

where $i \in 0,1,2, \ldots, N-1$. 


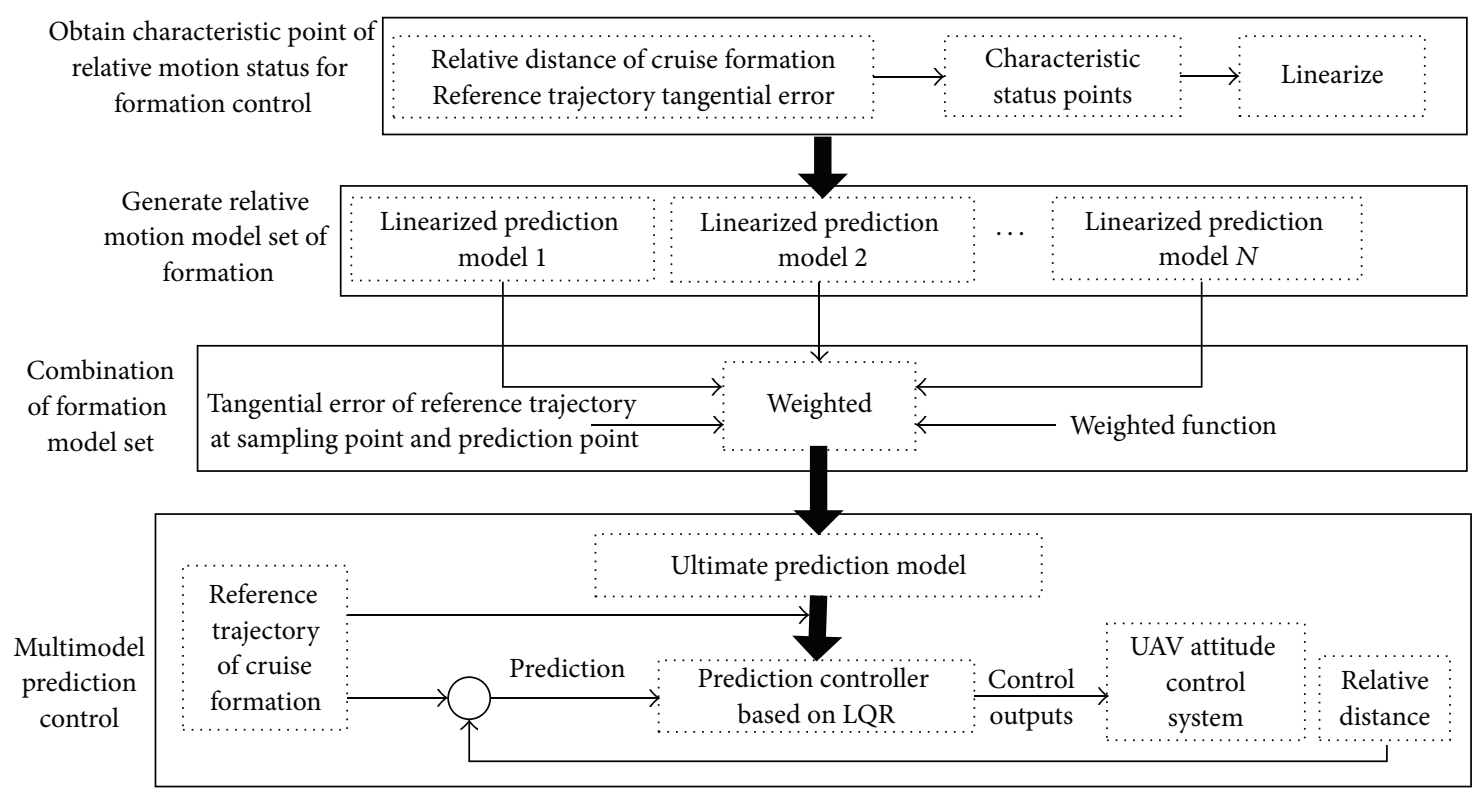

FIGURE 5: The schematic of multimodel control method for UAV formation flight.

After using multiple models, the performance index is linear quadratic, whose constraints are linear equality and inequality, so the optimization problem is a linear quadratic programming problem. The solution methods of linear quadratic programming problem can be used to solve the receding optimization problem. The linear quadratic programming problem is a common programming problem and has a lot of solution methods and higher speed than the ordinary nonlinear programming, which increases the speed of receding optimization solution [22].

\section{Simulation}

In this section, numerical simulations are performed to demonstrate the performance of the proposed approach. Here, the formation control ability can be tested in two important scenarios. Simulation scenarios are set as follows. One scenario is the leader UAV flying straight, and the other is the leader UAV flying with turning course. Additionally, the comparison simulation between single MPC (SMPC) method and multiple MPC (MMPC) method is carried on to verify effectiveness of the method in this paper. Meanwhile, the parameters used in the simulations are set as follows. The prediction horizon $N$ is 5 and the sampling interval is $0.2 \mathrm{~s}$. The angular velocity and velocity of two vehicles are confined during the interval $(-0.1,0.1)$ and the interval $(35,45)$, respectively. All the computations and experiments have been on a computer with Inter Core i3 CPU, $3.30 \mathrm{GHz}$, and Windows XP operating systems. Table 1 summarizes the initial conditions of the formation.

4.1. Formation Simulation of Leader UAV Flying Straight. The simulation experiment is mainly used to verify the UAV formation control capability when the leader UAV is flying straight. Here, error exists in the position measurement of
TABLE 1: Initial conditions of UAV formation.

\begin{tabular}{lcc}
\hline \multirow{2}{*}{ Initial conditions } & \multicolumn{2}{c}{ The role of UAV } \\
& Leader & Follower \\
\hline Initial position & $(0,0)$ & $(-100,-100)$ \\
Initial angle & 0 & $\pi / 2$ \\
Initial velocity & 40 & 40 \\
Initial angular velocity & 0 & 0 \\
\hline
\end{tabular}

leader UAV, which is $\pm 0.5 \mathrm{~m}$. There are two different control goals. One is that the relative position between follower and leader of UAV formation in the track coordinates is as follows:

$$
\begin{gathered}
X=-60, \\
Y=30
\end{gathered}
$$

The other is that the formation should be formed within $40 \mathrm{~s}$.

Because the leader UAV has its initial angle of $\pi / 4$ and it flies straight, the initial relative position in the track coordinates will be obtained as follows:

$$
\left[\begin{array}{l}
x_{d} \\
y_{d}
\end{array}\right]=\left[\begin{array}{cc}
\cos \frac{\pi}{4} & \sin \frac{\pi}{4} \\
-\sin \frac{\pi}{4} & \cos \frac{\pi}{4}
\end{array}\right]\left[\begin{array}{l}
-100 \\
-100
\end{array}\right]=\left[\begin{array}{c}
-100 \sqrt{2} \\
0
\end{array}\right] .
$$

Simulation is carried out by using Matlab Simulink toolbox, and the simulation results are shown from Figures 6, 7, 8, 9, and 10 .

According to Figures $6-10$, it can be seen that when leader UAV is navigating in a straight line, formation control can be achieved through both SMPC and MMPC method. However, the SMPC method has a larger tracking error than the MMPC method. Meanwhile, it can also be seen that it takes a longer time for SMPC method than MMPC method to 


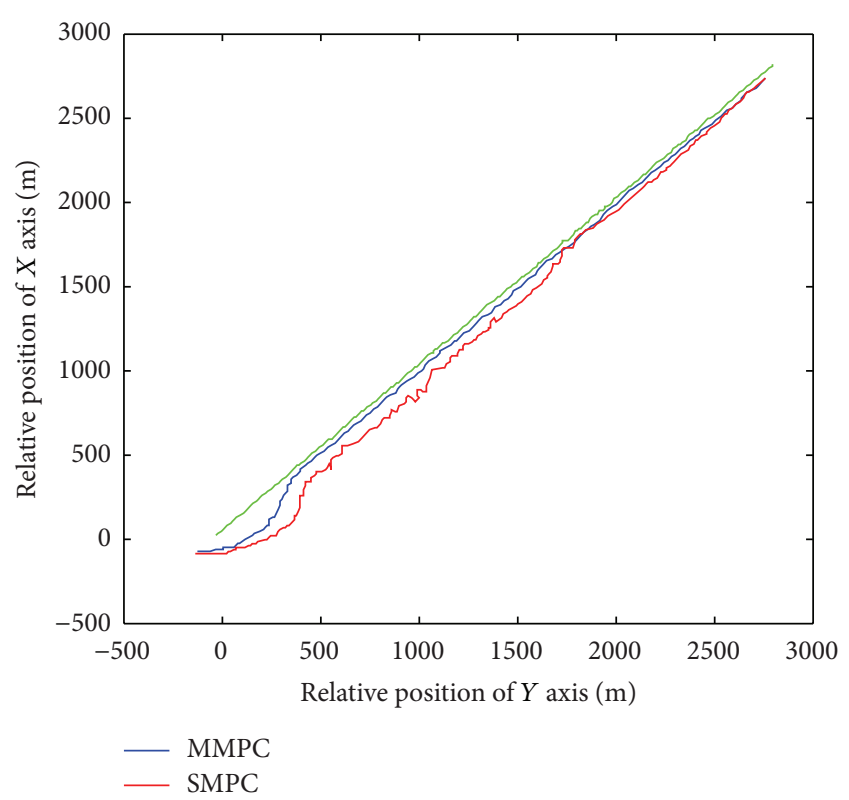

FIGURE 6: Flight trajectories of two UAVs.

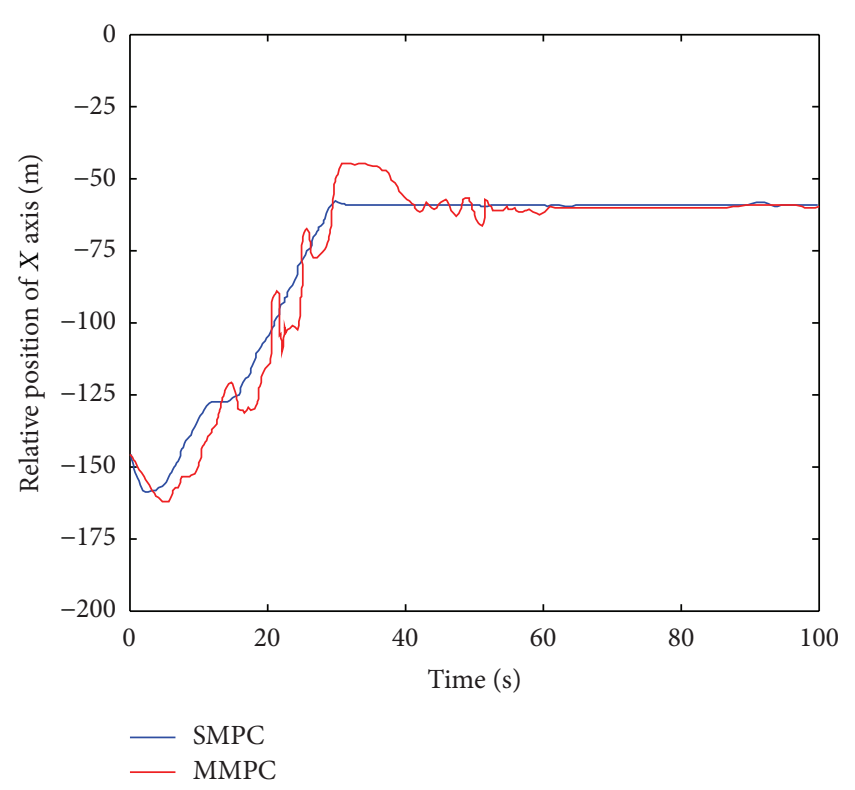

Figure 7: Relative position of $X$-axis.

form a steady formation. The UAV formation can be realized in 40 seconds by the MMPC method, which meets the actual design demand.

4.2. Formation Simulation for Leader UAV with Turning Flight. The UAV formation control capability is proved in this section when the leader UAV flies with a turning flight path. The UAV flies $20 \mathrm{~s}$ with an initial angle of $0^{\circ}$ between the leader UAV and $X$-axis, and then the UAV flies with angular velocity of $\pi / 200$ for 100 seconds, and then it moves straight

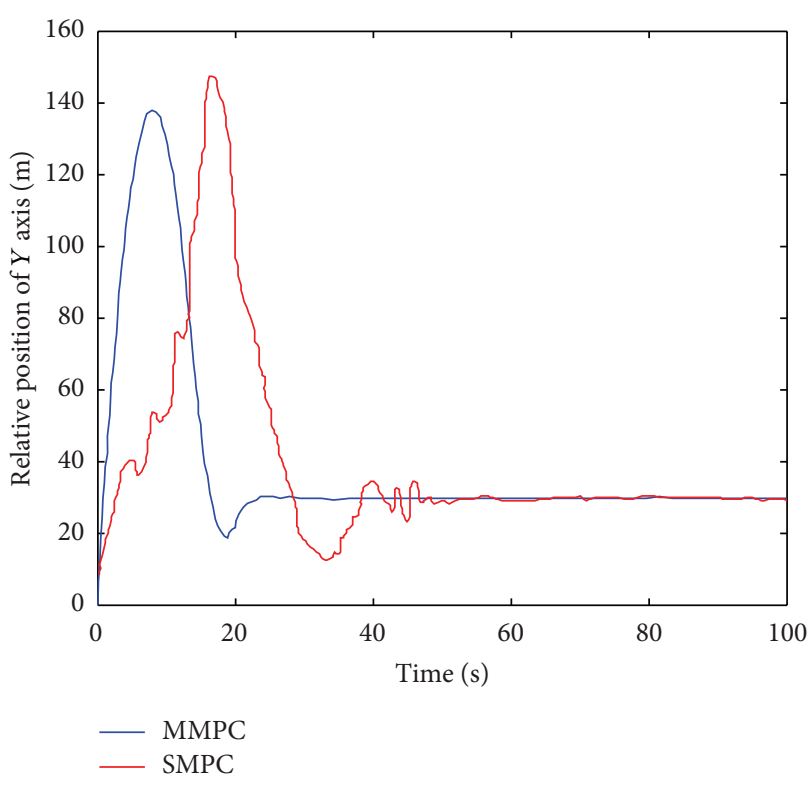

FIGURE 8: Relative position of $Y$-axis.

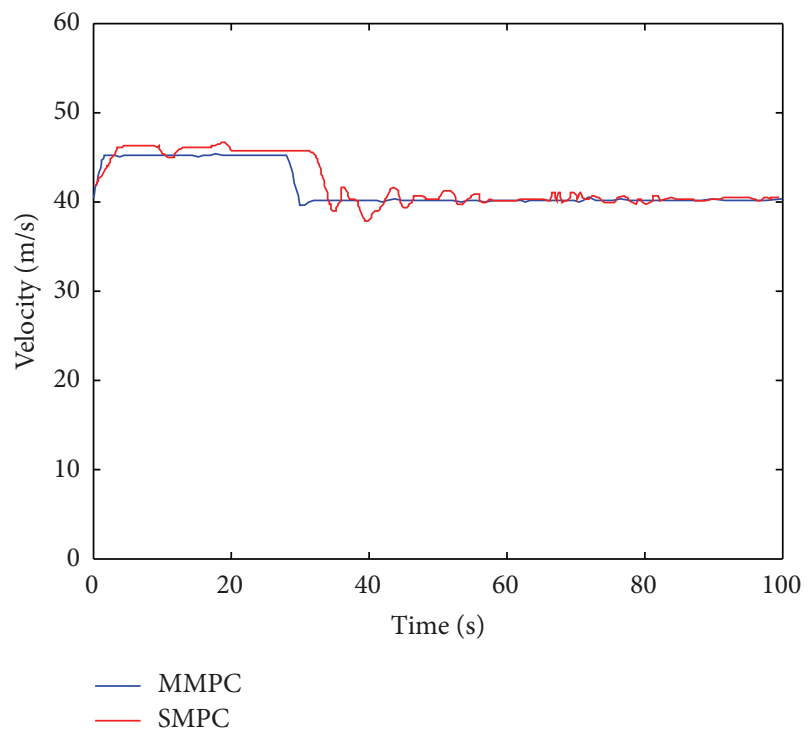

FIGURE 9: Change curve of follower UAV's velocity.

in $Y$-axis direction. There are also two different control goals. One is that the relative position between follower and leader of UAV formation in the track coordinates is as follows:

$$
\left[\begin{array}{c}
x_{\mathrm{dref}} \\
y_{\mathrm{dref}}
\end{array}\right]=\left[\begin{array}{l}
-50 \\
-50
\end{array}\right]
$$

The other is the formation should form within $40 \mathrm{~s}$.

From Table 1, the relative position in the track coordinate system between two vehicles is obtained as follows:

$$
\left[\begin{array}{l}
x_{d} \\
y_{d}
\end{array}\right]=\left[\begin{array}{cc}
\cos 0 & \sin 0 \\
-\sin 0 & \cos 0
\end{array}\right]\left[\begin{array}{l}
-100 \\
-100
\end{array}\right]=\left[\begin{array}{l}
-100 \\
-100
\end{array}\right] \text {. }
$$




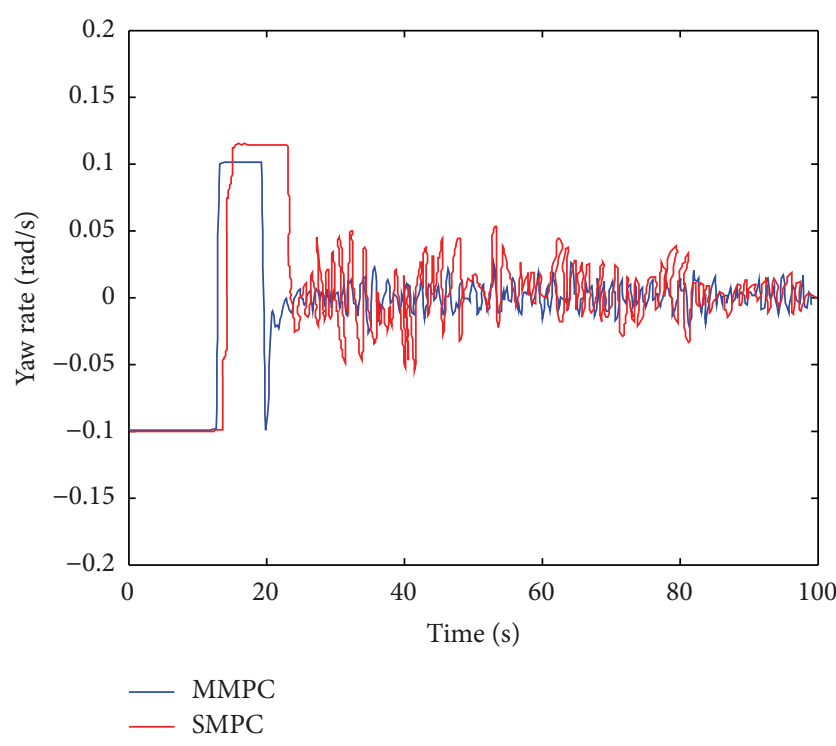

FIGURE 10: Change curve of follower UAV's yaw rate.

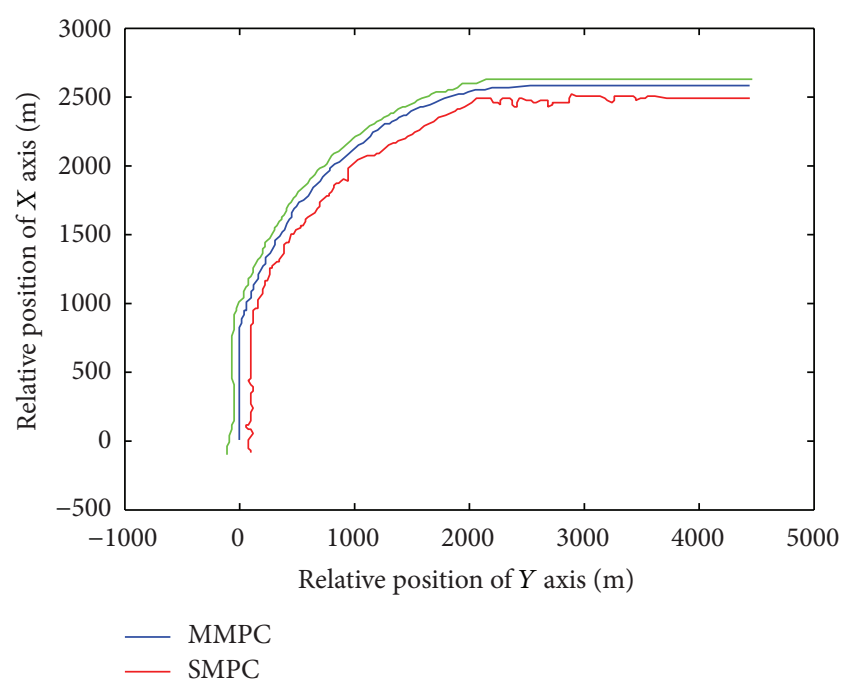

FIGURE 11: Flight trajectories of two UAVs.

Simulation is carried out by using Matlab Simulink toolbox, and the results are shown in Figures 11, 12, 13, 14, and 15.

According to Figures 10-14, when leader UAV flies with a turning flight path, using method proposed in the paper, formation control can be achieved better than the SMPC method, whenever the UAV flies straight or flies with a turning path. The SMPC method has a larger tracking error than the MMPC method. Meanwhile, it can also be seen that it takes a longer time for SMPC method than MMPC method to form a steady formation. The UAV formation can be realized in 40 seconds by the MMPC method, which meets the actual design demand.

According to the Matlab simulation process of UAV formation in those two scenarios above, when the sampling

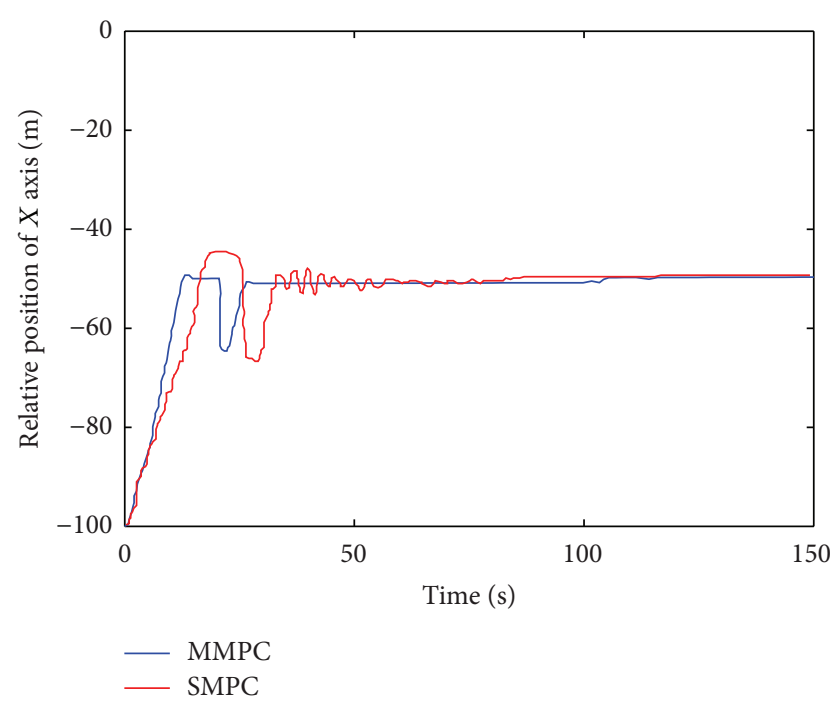

FIgURE 12: Relative position of $X$-axis.

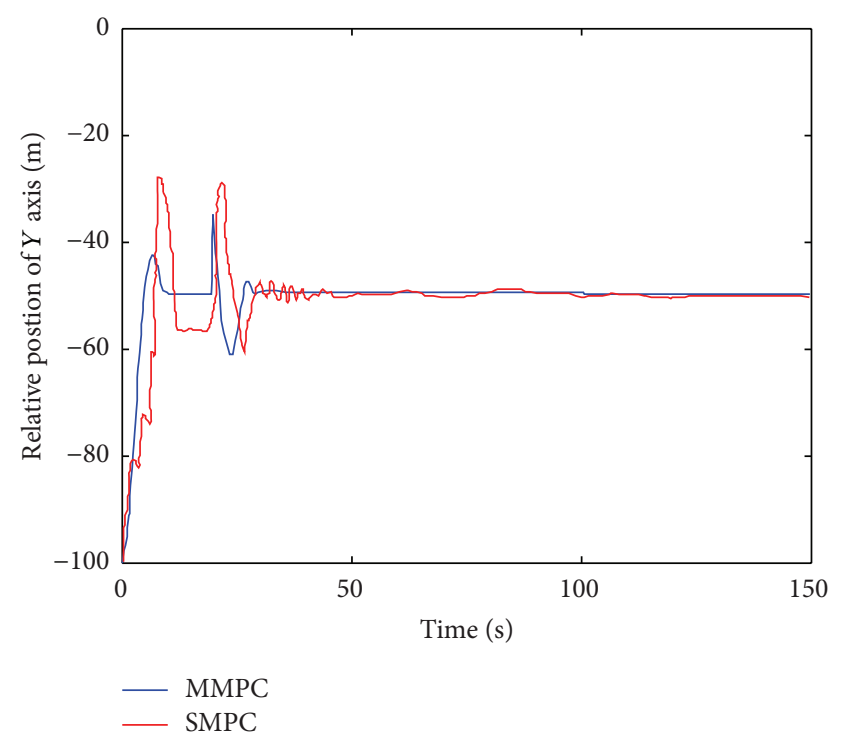

FIGURE 13: Relative position of $Y$-axis.

interval is $0.2 \mathrm{~s}$, the simulation time of the receding optimization program on the $\mathrm{PC}$ is less than $0.2 \mathrm{~s}$ each time and the time will be shorter if the simulation is done on a dedicated chip. So it meets the real-time needs. It can be seen from the relative position on the $X$-axis and $Y$-axis of two vehicles in the track coordinate system that the UAV formation is realized within $40 \mathrm{~s}$. All in all, the simulation shows that the control requirements and real-time requirements can be satisfied by using multimodel predictive control method for UAV formation control. 


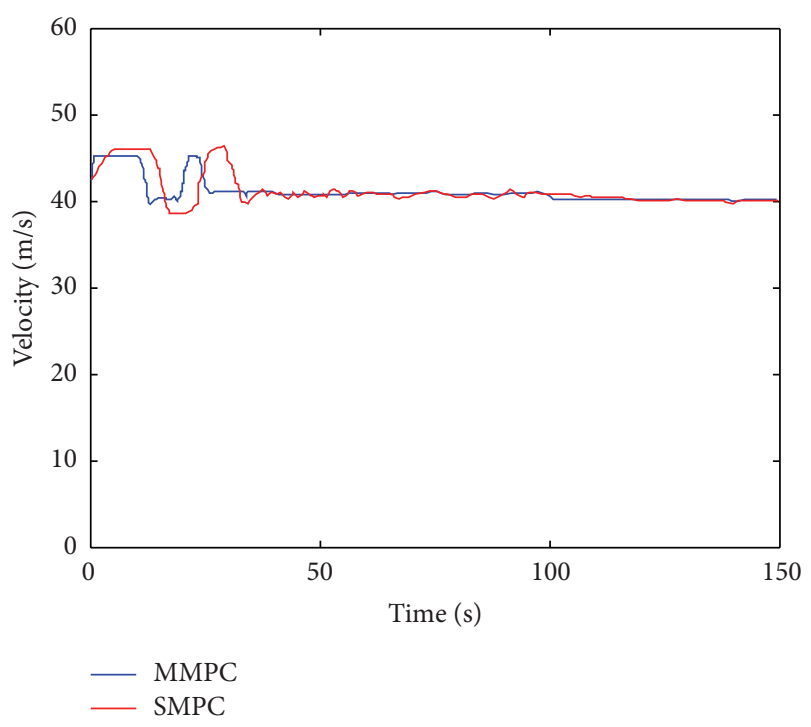

FIGURE 14: Change curve of follower UAV's velocity.

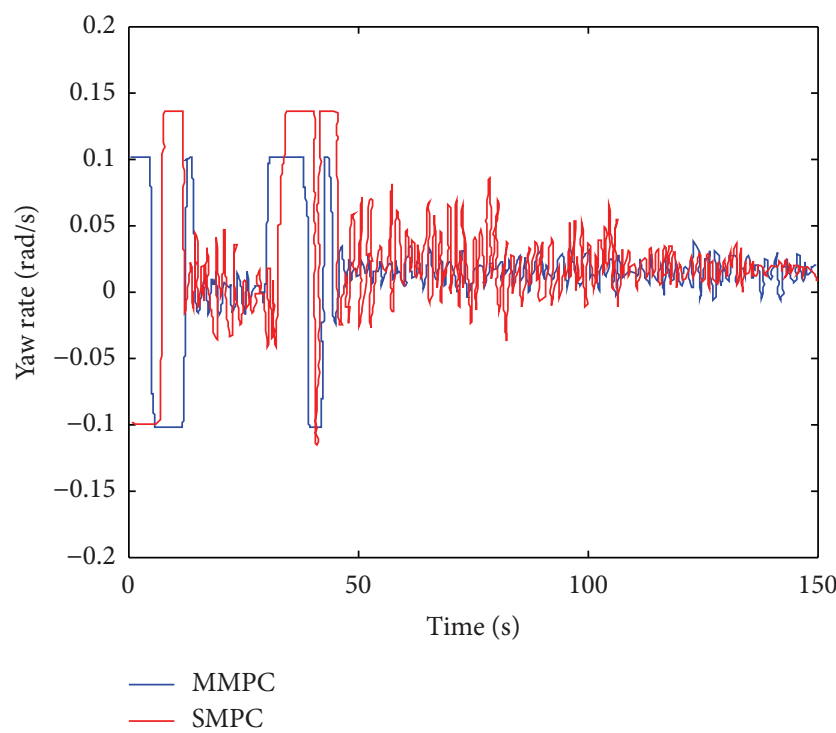

FIGURE 15: Change curve of follower UAV's yaw rate.

\section{Conclusion}

In this paper, the main work can be concluded as follows to solve the problem of UAV formation control.

(1) Discrete relative motion equations are established for UAV formation by using the leader-follower method.

(2) Multimodel sets for UAV formation are established, and the weighted model sets method is proposed.

(3) The formation controller based on multimodel predictive control is designed.

(4) Simulation in two scenarios is carried out and the effectiveness of controller designed and control strategy is verified.
The multimodel predictive control method can be used for UAV formation control. This method can meet control requirements and real-time requirements well. The result of this paper is the basis of further research on formation reconfiguration control problem. In the future, we will introduce the approach proposed in this paper to the controller design of actual UAV formation flight.

\section{Conflict of Interests}

The authors declare that there is no conflict of interests regarding the publication of this paper.

\section{Acknowledgment}

Research is supported by the National Science Foundation of China (NSFC) under Grants no. 51201182 and 61105012, and National Aviation Science Foundation of China (NASFC) under Grant no. 20135896027. Among these foundations, the NASFC is a cooperation program of our research group and FACRI, and this foundation requires both sides to publish an article.

\section{References}

[1] X.-Y. Wang, X.-M. Wang, and C.-C. Yao, "Design of UAVs formation flight controller based on neural network adaptive inversion," Control and Decision, vol. 28, no. 6, pp. 837-843, 2013.

[2] C.-J. Ru, R.-X. Wei, J. Dai, D. Shen, and L.-P. Zhang, "Autonomous reconfiguration control method for UAV's formation based on Nash bargain," Auta Automatica Sinica, vol. 39, no. 8, pp. 1349-1359, 2013.

[3] L. Jieun, S. K. Hyeong, and K. Youdan, "Formation geometry center based formation controller design using Lyapunov stability theory," KSAS International Journal, no. 2, pp. 71-76, 2008.

[4] A. Bemporad and C. Rocchi, "Decentralized hybrid model predictive control of a formation of unmanned aerial vehicles," in Proceedings of the 18th IFAC Word Congress, Milanno, Italy, 2011.

[5] Z. Chao, S.-L. Zhou, L. Ming, and W.-G. Zhang, "UAV formation flight based on nonlinear model predictive control," Mathematical Problems in Engineering, vol. 2012, Article ID 261367, 15 pages, 2012.

[6] K. Wesselowski and R. Fierro, "A dual-mode model predictive controller for robot formations," in Proceedings of the 42nd IEEE Conference on Decision and Control, pp. 3615-3620, Maui, Hawaii, USA, December 2003.

[7] B. J. N. Guerreiro, C. Silvestre, and R. Cunha, "Terrain avoidance nonlinear model predictive control for autonomous rotorcraft," Journal of Intelligent \& Robotic Systems: Theory and Applications, vol. 68, no. 9, pp. 69-85, 2012.

[8] M. A. Abbas, J. M. Eklund, and R. Milman, "Real-time analysis for nonlinear model predictive control of autonomous vehicles," in Proceedings of the 25th IEEE Canadian Conference on Electrical \& Computer Engineering (CCECE '12), pp. 1-4, 2012.

[9] J. Shin and H. J. Kim, "Nonlinear model predictive formation flight," IEEE Transactions on Systems, Man, and Cybernetics A: Systems and Humans, vol. 39, no. 5, pp. 1116-1125, 2009. 
[10] F. Alessandro, L. Sauro, and M. Andrea, "Nonlinear decentralized model predictive control strategy for a formation of unmanned aerial vehicles," in Proceedings of the 2nd IFAC Workshop on Multivehicle System, vol. 2, pp. 49-54, 2012.

[11] C. Gorman and N. Slegers, "Predictive control of general nonlinear systems using series approximations," in Proceedings of the AIAA Guidance, Navigation, and Control Conference and Exhibit, AIAA 2009-5994, Chicago, Ill, USA, August 2009.

[12] W. Dhouib, M. Djemel, and M. Chtourou, "Fuzzy predictive control of nonlinear systems," in Proceedings of the 8th International Multi-Conference on Systems, Signals and Devices (SSD '11), pp. 1-8, Sousse, Tunisia, March 2011.

[13] T. Keviczky, F. Borrelli, and G. J. Balas, "Decentralized receding horizon control for large scale dynamically decoupled systems," Automatica, vol. 42, no. 12, pp. 2105-2115, 2006.

[14] Q. Chen, L. Gao, R. A. Dougal, and S. Quan, "Multiple model predictive control for a hybrid proton exchange membrane fuel cell system," Journal of Power Sources, vol. 191, no. 2, pp. 473482, 2009.

[15] N. N. Nandola and S. Bhartiya, "A multiple model approach for predictive control of nonlinear hybrid systems," Journal of Process Control, vol. 18, no. 2, pp. 131-148, 2008.

[16] D. Dougherty and D. Cooper, "A practical multiple model adaptive strategy for single-loop MPC," Control Engineering Practice, vol. 11, no. 2, pp. 141-159, 2003.

[17] K. S. Narendra and C. Xiang, "Adaptive control of discretetime systems using multiple models," IEEE Transactions on Automatic Control, vol. 45, no. 9, pp. 1669-1686, 2000.

[18] L.-L. Liu, L.-F. Zhou, T. Ji, and Y.-H. Zhao, "Research on model switching method of multi-hierarchical model predictive control systems," Acta Automatica Sinica, vol. 39, no. 5, pp. 626630, 2013.

[19] R. J. Spiegel, M. W. Turner, and V. E. McCormick, "Fuzzylogic-based controllers for efficiency optimization of inverterfed induction motor drives," Fuzzy Sets and Systems, vol. 137, no. 3, pp. 387-401, 2003.

[20] Z.-Q. Chen and H.-M. Jiang, "T-S fuzzy model predictive control simulation based on intelligent optimization algorithm," Journal of System Simulation, vol. 2, pp. 79-85, 2009.

[21] Y. Gu, H. O. Wang, K. Tanaka, and L. G. Bushnell, "Fuzzy control of nonlinear time-delay systems: stability and design issues," in Proceedings of the American Control Conference (ACC '01), pp. 4771-4776, Arlington, Calif, USA, June 2001.

[22] T. Keviczky, F. Borrelli, K. Fregene, D. Godbole, and G. J. Balas, "Decentralized receding horizon control and coordination of autonomous vehicle formations," IEEE Transactions on Control Systems Technology, vol. 16, no. 1, pp. 19-33, 2008. 


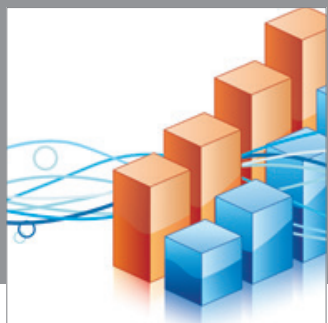

Advances in

Operations Research

mansans

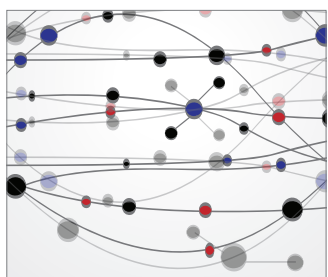

The Scientific World Journal
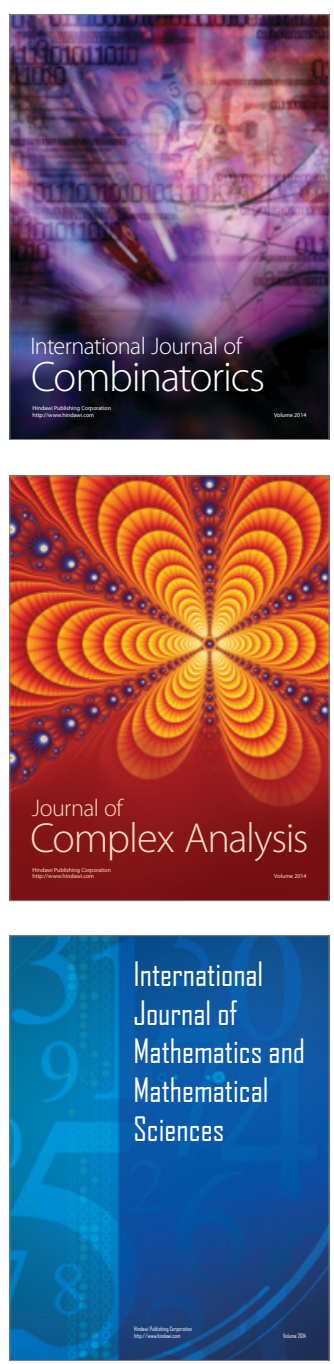
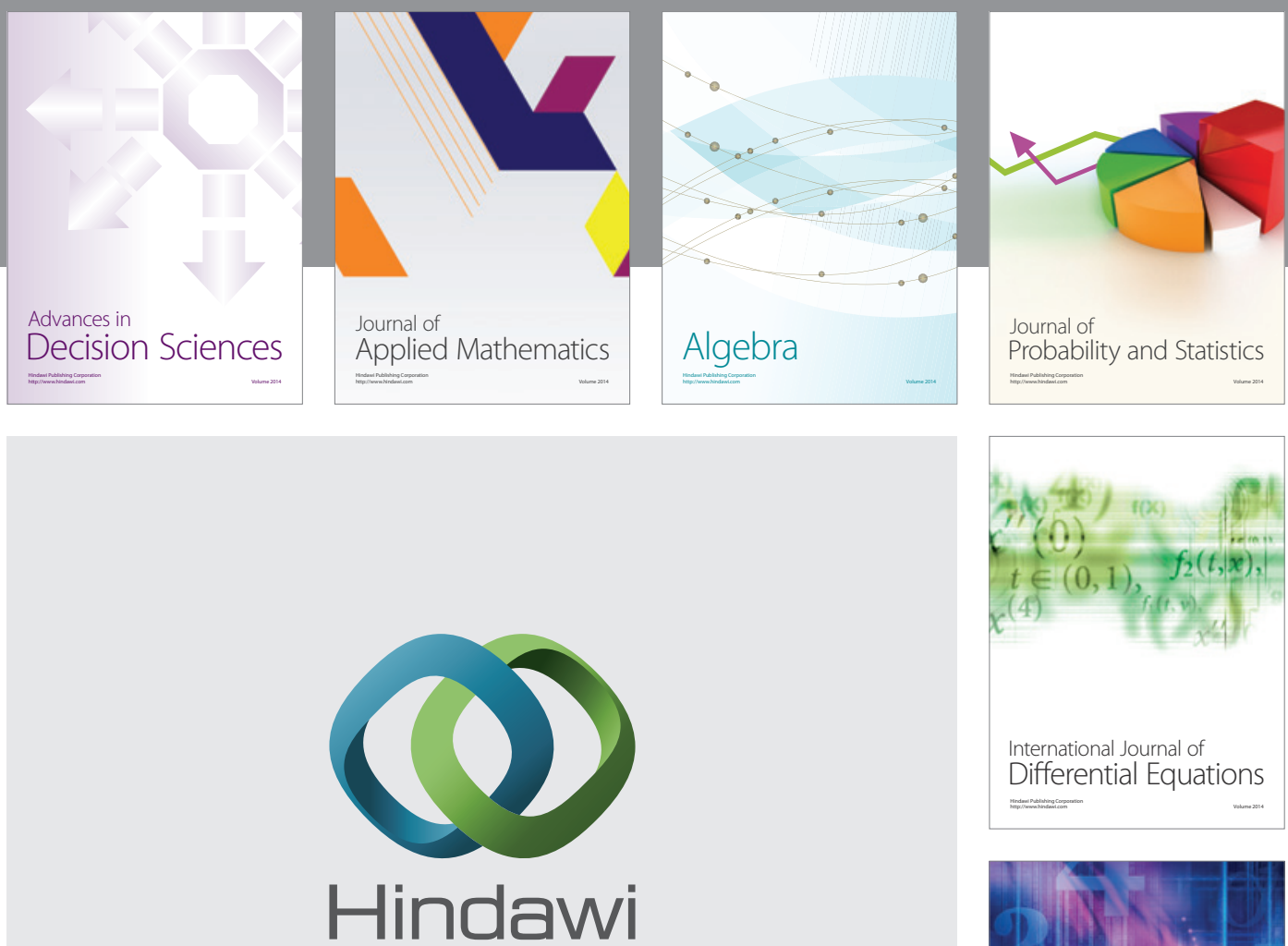

Submit your manuscripts at http://www.hindawi.com
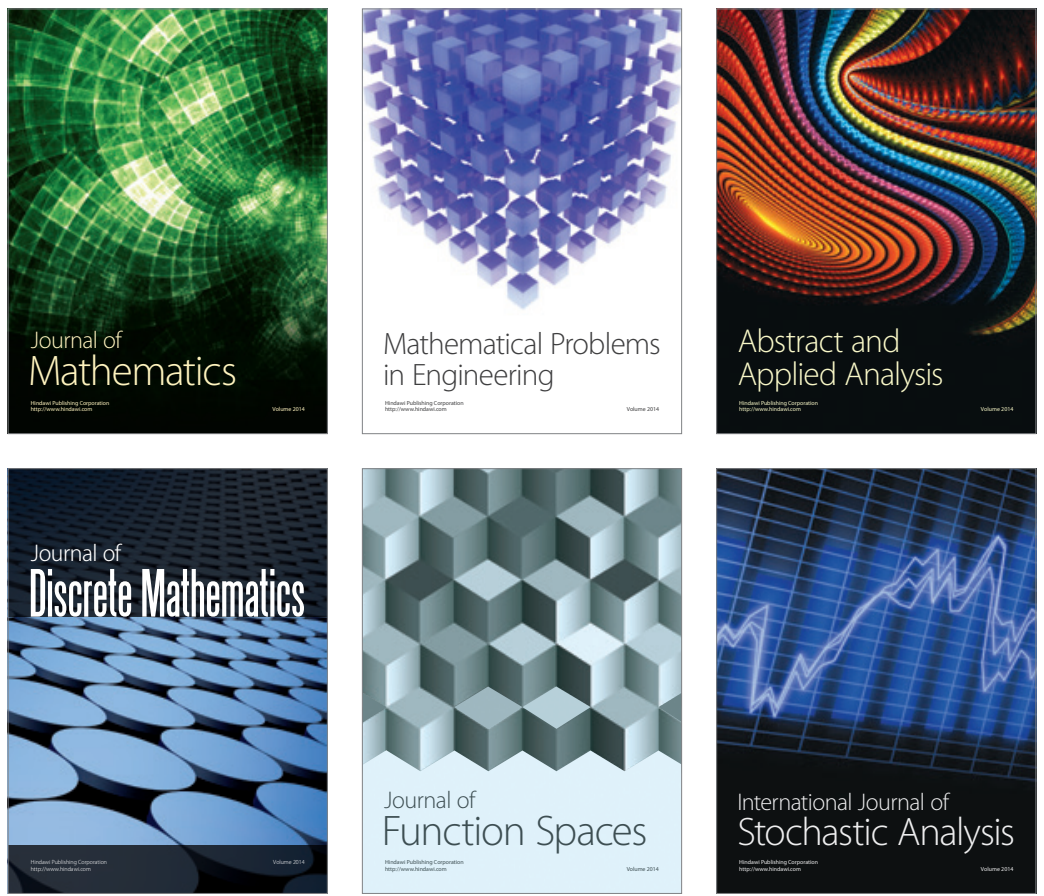

Journal of

Function Spaces

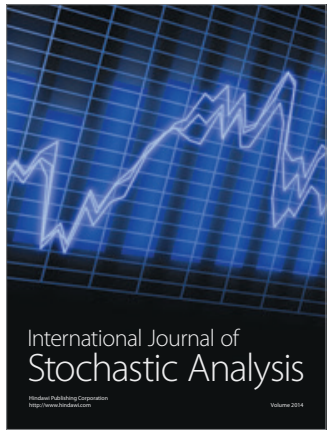

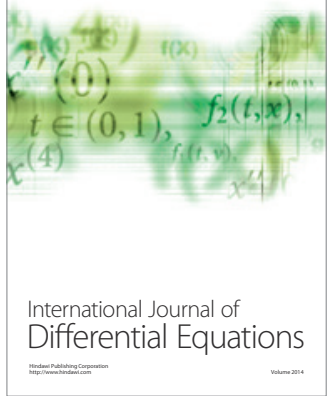
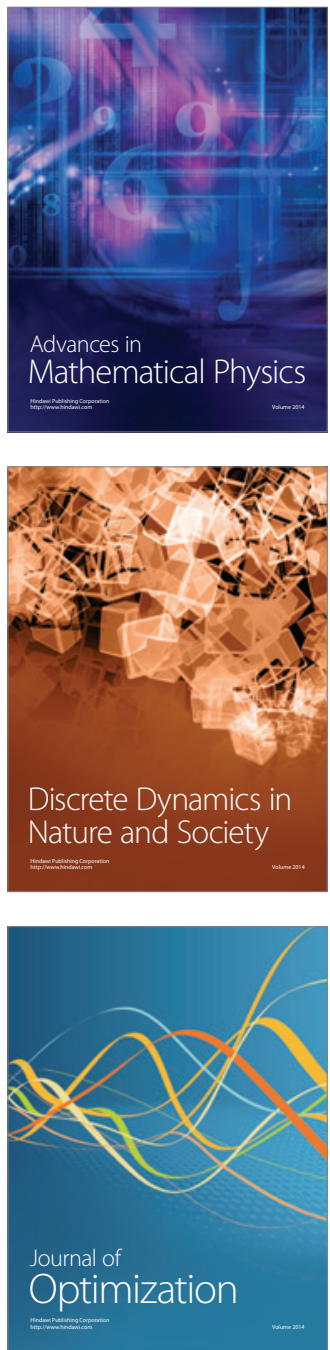